Ocular Oncology

and Pathology
Ocul Oncol Pathol 2017;3:110-112

DOI: $10.1159 / 000451058$
Received: June 27, 2016

Accepted after revision: September 16, 2016

Published online: November 9, 2016

\title{
Optic Disc Pyogenic Granuloma
}

\author{
Eduardo F. Marback Luciano Espinheira Fonseca Jr. Roberto L. Marback \\ Federal University of Bahia, Salvador da Bahia, Brazil
}

\section{Key Words}

Orbit · Metastasis · Orbital tumor · Adenoid cystic carcinoma of the lacrimal gland - Adenoid cystic carcinoma .

Lacrimal gland carcinoma

\begin{abstract}
Aim: The aim of this paper was to report a rare presentation of adenoid cystic carcinoma. Method: This is a case report presenting clinical, radiographic, and histopathologic findings. Results: A 72-year-old female with no reported ocular symptoms was found to have an incidental right orbital mass on imaging. Additional studies revealed multiple liver lesions, which were biopsied and found to be consistent with adenoid cystic carcinoma. She was then referred to the ophthalmology service, where ocular examination demonstrated $2.5 \mathrm{~mm}$ of right proptosis with elevation and abduction deficits. Diplopia could be elicited in extreme upgaze and right lateral gaze. An excisional biopsy of the orbital mass was performed, with histopathology confirming the diagnosis of primary adenoid cystic carcinoma of the lacrimal gland, thereby also supporting the initial suspicion that the hepatic adenoid cystic carcinoma lesions represented metastases. Conclusion: The authors describe a rare presentation of adenoid cystic carcinoma of the lacrimal gland, initially asymptomatic, with metastatic lesions restricted to the liver at the time of diagnosis. Three previous cases of adenoid cystic car-
\end{abstract}

cinoma with isolated metastatic hepatic lesions at the time of diagnosis have been reported; all of these cases localized the primary tumor to the salivary glands.

(C) 2016 S. Karger AG, Basel

\section{Introduction}

Pyogenic granuloma (PG) is a benign vascular lesion probably of reactive nature $[1,2]$. It usually occurs in the skin and mucous membranes. Ophthalmologists are familiar with its occurrence in the conjunctiva, although sometimes it can be seen in the cornea or lacrimal drainage system $[1,2]$.

We report an unusual case of an optic disc PG referred as an intraocular melanoma in a blind, painful eye.

\section{Case Presentation}

A 63-year-old diabetic female was referred for ocular oncology evaluation after being admitted to the hospital for a possible globe evisceration procedure with endophthalmitis due to a perforated corneal ulcer in the right eye. She reported complete vision loss in the right eye many years before due to uncontrolled glaucoma. Examination revealed no light perception, diffuse conjunctival hyperemia, and a wide corneal defect with haze media precluding fundus observation (Fig. 1a). On ultrasonography, a 5.0-mm thick, pedunculated, solid lesion first interpreted as mushroom-shaped

\section{KARGER}

(C) 2016 S. Karger AG, Basel

E-Mail karger@karger.com

www.karger.com/oop
Eduardo F. Marback, MD, PhD

Rua Eduardo Jose dos Santos, 147, Sala 806

Salvador da Bahia, BA 41940-455 (Brazil)

E-Mail eduardomarback@uol.com.br 

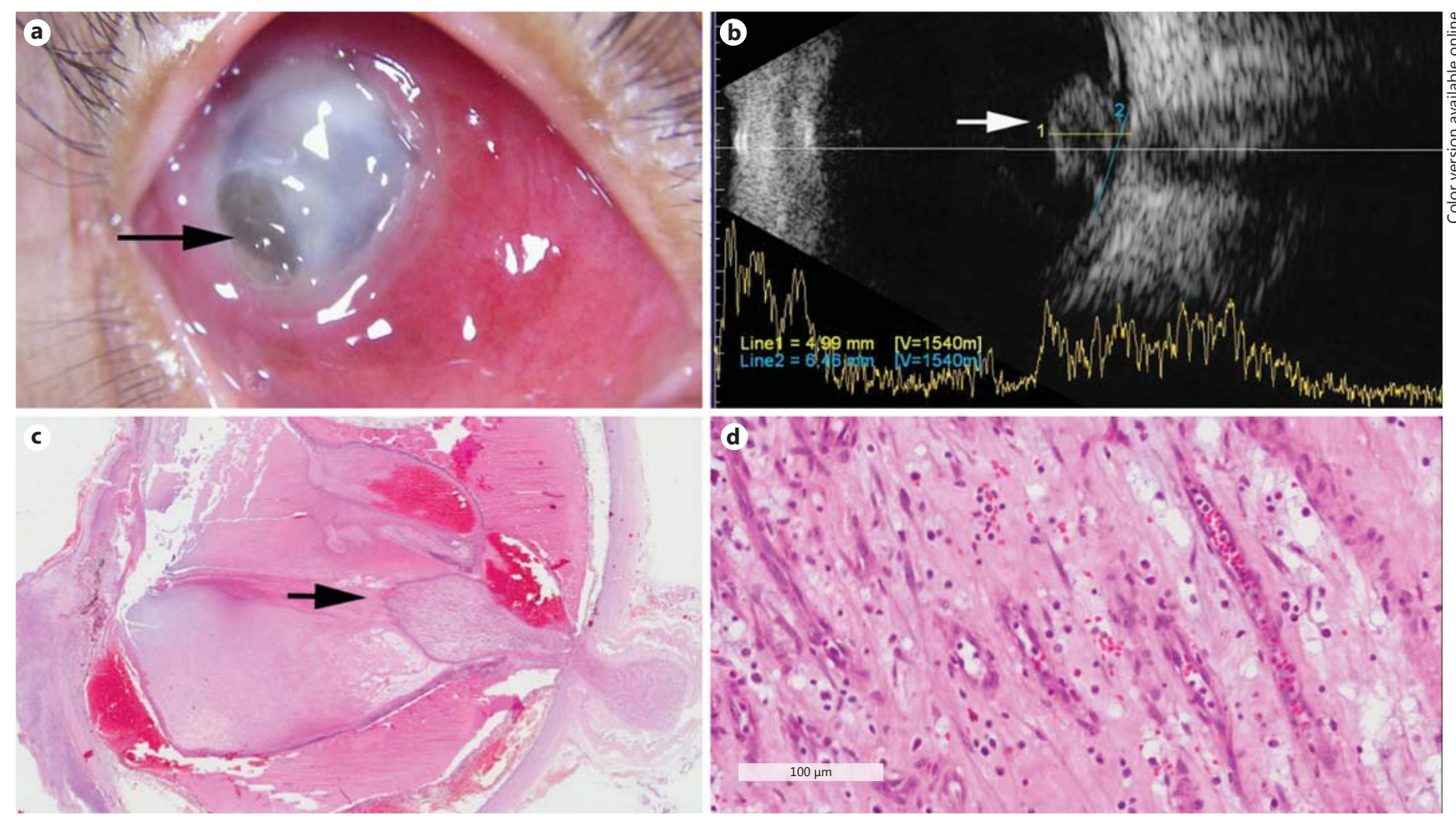

Fig. 1. a Clinical picture. Diffuse conjunctival congestion and corneal melting with large perforation (arrow). b Ultrasound of the mushroom-shaped lesion (arrow) with an internal acoustic quiet zone. c Pedunculated lesion (granulation tissue) emerging from the optic disc $(\mathrm{H} \& \mathrm{E})$. d Granulation tissue; capillaries in a loose stroma with inflammatory cell infiltration. H\&E. $\times 400$.

tumor was identified, covering part of the optic disc. The lesion showed an internal acoustic quiet zone, spontaneous vascular pulsations, and floating particles in the vitreous (Fig. 1b). There was associated retinal detachment and sparse vitreous particulate echoes. Based on clinical suspicion of choroidal melanoma, the eye was enucleated.

Histopathological evaluation revealed corneal perforation with iris incarceration. The anterior chamber was shallow and filled with polymorphonuclear inflammatory cells. The retina was detached, and the vitreous exhibited a prominent abscess. A mass with a typical "Christmas tree" shape was noted emerging from the optic disc. The pedunculated mass lesion was composed of many small vascular channels into a loose myxoid stroma with inflammatory infiltration mainly composed of neutrophils and lymphocytes (Fig. 1c, d).

\section{Discussion}

Many authors consider PG to be a lobular capillary hemangioma. Stagner and Jakobiec [2] argue against this classification, since lobular capillary hemangioma usually is an ulcerated skin or mucosal tumor that occurs in children and pregnant women, with no relationship to trauma or other inflammatory triggers. In the eye and ocular adnexa, PG seems to be a reactional lesion that occurs almost exclusively in the conjunctiva and rarely in the cornea and lacrimal drainage apparatus [1]. It is usually related to incidental trauma, chalazion, retinal surgery, lacrimal drainage surgery, lacrimal punctum plugs, orbital implants, pterygium surgery, eyelid surgery, strabismus surgery, and it sometimes occurs spontaneously [1, 3-6].

In the present case, optic disc PG simulated juxtapapillary melanoma in an eye with no possible fundus view. When clinicians are faced with blind eyes with opaque media, ophthalmologists generally rely on ultrasonography to guide their clinical diagnosis and management. Typical ultrasonographic findings of very high diagnostic accuracy have been reported for choroidal melanoma: mushroom shape for tumors that have broken through Bruch's membrane, an internal acoustic quiet zone, cho- 
roidal excavation, vascular pulsations, and medium-tolow internal reflectivity with $\kappa$ angle formation on A scan $[7,8]$. In our case, a pedunculated PG mimicked the typical uveal juxtapapillary mushroom-shaped melanoma. This shape was a simulation, since the mass was in reality a pedunculated lesion compressed by intraocular pressure, as could be seen on the macroscopic slide view (Fig. 1c) where the tumor and retinal detachment assumed a much more anterior location than the one seen on ultrasonography. B scan evaluation also revealed an internal acoustic quiet zone and spontaneous vascular pulsations. These features were suggestive of uveal melanoma [7] and prompted a wrong clinical suspicion. It is worthy to mention that vascular lesions usually exhibit high-reflectivity A scan with a brilliant internal aspect in B scan. In PG, the vascular component is predominantly formed by capillaries, and the stoma is loose with multiple inflammatory cells. We think that this histologic aspect would be compatible with the internal acoustic quiet zone seen in this case.

More than 50 distinct lesions have been reported to mimic primary uveal melanoma, and half of those were choroidal nevi $[8,9]$. Another important group that rep- resents over $20 \%$ of the cases is the hemorrhagic lesions such as peripheral exudative chorioretinopathy, age-related macular degeneration, suprachoroidal hemorrhage, polypoidal choroidal vasculopathy, and hemorrhagic retinal detachment [9].

In summary, we presented a case of optic disc PG in a blind, painful eye with a perforated corneal ulcer and endophthalmitis. Ultrasonography was highly suspicious of juxtapapillary melanoma. Although a lot of uveal pseudomelanomas have been reported, our literature search failed to find a similar case.

\section{Statement of Ethics}

The subject has given prior informed consent for the treatment and this paper.

\section{Disclosure Statement}

The authors declare that there are no conflicts of interest and no financial interests.

\section{References}

1 Ferry AP: Pyogenic granulomas of the eye and ocular adnexa: a study of 100 cases. Trans Am Ophthalmol Soc 1989;87:327-343.

2 Stagner AM, Jakobiec FA: A critical analysis of eleven periocular lobular capillary hemangiomas in adults. Am J Ophthalmol 2016;165: 164-173.

3 Kim BM, Osmanovic SS, Edward DP: Pyogenic granulomas after silicone punctal plugs: a clinical and histopathologic study. Am J Ophthalmol 2005;139:678-684.

4 Espinoza GM, Lueder GT: Conjunctival pyogenic granulomas after strabismus surgery. Ophthalmology 2005;112:1283-1286.
5 Owji N, Sadeghipour A, Salour H, Sahraeyan $\mathrm{N}$ : Pyogenic granuloma as a presenting sign of hydroxyapatite orbital implant exposure: a clinicopathologic study. Ophthal Plast Reconstr Surg 2006;22:467-471.

6 Ben Simon GJ, Molina M, Schwarcz RM, McCann JD, Goldberg RA: External (subciliary) vs internal (transconjunctival) involutional entropion repair. Am J Ophthalmol 2005;139: 482-487.
7 Collaborative Ocular Melanoma Study Group; Boldt HC, Byrne SF, Gilson MM, Finger PT, Green RL, Straatsma BR, Simpson ER, Hawkins BS: Baseline echographic characteristics of tumors in eyes of patients enrolled in the Collaborative Ocular Melanoma Study: COMS report no. 29. Ophthalmology 2008; 115:1390-1397.

8 Accuracy of diagnosis of choroidal melanomas in the Collaborative Ocular Melanoma Study. COMS report no. 1. Arch Ophthalmol 1990;108:1268-1273.

9 Shields JA, Mashayekhi A, Ra S, Shields CL: Pseudomelanomas of the posterior uveal tract: the 2006 Taylor R. Smith Lecture. Retina 2005;25:767-771. 